\title{
Exotic meson decays and polarization asymmetry in hadron en- vironment with chiral imbalance
}

\author{
A.E. Putilova ${ }^{1, \star}$ A.V. lakubovich ${ }^{1, \star \star}$ A.A. Andrianov ${ }^{1,2, \star \star \star}$ V.A. Andrianov ${ }^{1, \star \star \star \star}$ and D. Espriu ${ }^{2, \dagger}$ \\ ${ }^{1}$ Faculty of Physics, Saint Petersburg State University, Universitetskaya nab. 7/9, Saint Petersburg 199034, \\ Russia, and \\ ${ }^{2}$ Departament de Física Quàntica i Astrofísica and Institut de Ciéncies del Cosmos (ICCUB), Universitat de \\ Barcelona, Martí i Franqués 1, 08028 Barcelona, Spain
}

\begin{abstract}
The possibility of the formation of a local parity breaking in a quark-hadron medium is described as a result of a violation of chiral symmetry, i.e, the difference between the average numbers of right and left -handing quarks in the fireball after HIC.The phenomenology of LPB in the fireball induces the introduction of a topological charge and, accordingly, a topological (chiral) chemical potential. Using the effective meson Lagrangian motivated by QCD in the chiral medium the properties of light scalar and pseudoscalar mesons are analyzed. It is establish that exotic decays of scalar mesons arise as a result of mixing of $\pi$ and $a_{0}$ vacuum states in the presence of chiral imbalance. The pion electromagnetic formfactor obtains a parity-odd supplement which generates a photon polarization asymmetry in pion polarizability. We consider that the above-mentioned properties of LPB can be revealed in experiments on LHC, RHIC, CBM FAIR and NICA accelerators.
\end{abstract}

\section{Motivation and aims}

The report concerns the problem of CP- violation in the strong interactions in heavy-ion collisions (HIC) at high energies. In particular, we are interested in the generation of Local Parity Breaking (LPB) at the volume of the fireball with a chiral quark-hadron matter namely, the presence of Chirality Imbalance i.e. the difference between the average numbers of right and left- handed quarks. After hadronization of quark-hadron medium, the fireball mostly consists of the light mesons gas. In such medium mesons composed from nearly massless $u$ and $d$ quarks change their properties, which differ from vacuum ones. In particular, in heavy-ion collisions at high energies, with raising temperatures and baryon densities, a metastable state can appear in the fireball with a non-trivial topological/axial charge, which is related to the gluon gauge field. As described earlier [1] in such an environment is possible the emergence of a gradient density of isosinglet pseudoscalar condensate which can be formed as a result of large, "long-lived" topological fluctuations of gluon fields in the fireball in central

\footnotetext{
^e-mail: alput@gmail.com

$\star \star$ e-mail: a.iakubovich@gmail.com

$\star \star \star$ e-mail: andrianov@icc.ub.edu

$\star \star \star \star$ e-mail: v.andriano@ rambler.ru

†e-mail: espriu@icc.ub.edu
} 
collisions [1]. To describe in fireball such a quark-hadron matter with chiral imbalance we must introduce the axial/chiral chemical potential [1]. Moreover, there are some experimental indications of an abnormal dilepton excess in the range of low invariant masses and rapidities, and moderate values of the transverse momenta [2], which can be partially thought of as a result of LPB in the medium (the details can be found in [3]).

We are going to investigate new properties of the light mesons in a chiral imbalance medium. The work includes the following items: finding of the modified vertices of meson decays; obtaining of the decay widths dependence on energy and chiral chemical potential; consideration of a pion-photon scattering in chiral medium; definition and calculation an expression for the polarization asymmetry in a pion-photon scattering.

\section{The chiral imbalance in medium and axial chemical potential}

Parity is one of the well established global symmetries of strong interactions. While there are arguments to think that parity cannot be broken in the usual QCD vacuum, this may not be the case under extreme conditions of temperature and density. The possibility of pion-condensation at a certain density, as the onset of an inhomogeneous classical pion field in the ground state of nuclear matter going back to the work of Migdal [4].

However, more recent and more complete effective theory studies [3] indicate that such a phase is a real possibility at moderate densities (from 3 up to 8 nuclei density). This possibility is relevant in astrophysical contexts but it may still be possible to produce such a phase in particle accelerators in conditions where the density is high. It is equivalent to a large baryonic chemical potential and low temperature. These conditions may be within reach of future experiments at FAIR and NICA.

We would like to consider the possibility that in a collision it could be possible to produce longlived bubbles with a non-vanishing value of the topological charge, where parity is locally broken. These topological fluctuations would take place in a finite volume and large temperature and these topological fluctuations were first proposed by Kharzeev, Pisarski and Tytgat [5] and later considered in a number of works [6-8]. Large fluctuations in the gauge field could exist in a hot environment and could generate a local chiral imbalance quarks which is associated with topological charge. This picture is supported by lattice simulations [9] although results obtained in euclidean simulations are hard to connect with the time-dependent dynamics that exist in the early stages of heavy ion collisions.

If a topological charge arises in a finite volume due to quantum fluctuations and it is conserved for a sufficiently long time in a quasi-equilibrium situation, we can introduce a topological chemical potential. The violation of the group $U(1)_{A}$ in quantum chromodynamics leads to the formula

$$
\partial^{\mu} J_{5, \mu}=2 i \bar{\psi} \hat{m}_{q} \gamma_{5} \psi+\frac{N_{f} g^{2}}{8 \pi^{2}} \epsilon_{\mu \nu \alpha \beta} \operatorname{tr}\left(G_{\mu \nu} G_{\alpha \beta}\right)
$$

where $J_{5, \mu}$ chiral current, $\hat{m}_{q}$ quark matrix, $\psi$ quark field, $G_{\mu \nu}$ gluon field, $g$ constant of strong interaction, $N_{f}$ number of quark flavours. The contribution of instantons, sphalerons, or other topological solutions is given by formula

$$
\mathcal{L}_{\theta}=\theta \frac{g^{2}}{16 \pi^{2}} \epsilon_{\mu \nu \alpha \beta} \operatorname{tr}\left(G_{\mu \nu} G_{\alpha \beta}\right)
$$

In the standard quantum chromodynamics, the angle $\theta$ is assumed as a constant and a very small quantity. We consider that in the medium $\theta$ is not small and is some pseudoscalar field. Using (1) and (2), we can obtain

$$
\mathcal{L}_{\theta}=\frac{-1}{2 N_{f}} J_{5, \mu} \partial^{\mu} \theta(x)-\frac{\theta(x)}{N_{f}} i \bar{\psi} \hat{m}_{q} \gamma_{5} \psi
$$


Further, for simplicity, we assume that $\theta$ depends only on time like that the time derivative of $\theta$ is simply a space-time constant, which we will call the chiral chemical potential $\mu_{5}$ :

$$
\frac{1}{2 N_{f}} \partial^{0} \theta(t)=\mu_{5}=\text { const } .
$$

Thus we have

$$
\left\langle\Delta T_{5}\right\rangle \simeq \frac{1}{2 N_{f}}\left\langle Q_{5}^{q}\right\rangle \Longleftrightarrow \mu_{5} \simeq \frac{1}{2 N_{f}} \mu_{\theta},
$$

Thus adding to the QCD lagrangian the term $\Delta \mathcal{L}_{\text {top }}=\mu_{\theta} \Delta T_{5}$ or $\Delta \mathcal{L}_{q}=\mu_{5} Q_{5}^{q}$, we get the possibility of accounting for non-trivial topological fluctuations (fluctons) in the nuclear (quark) fireball. In the Lorentz invariant form the field dual to fluctons is described by means of the classical pseudoscalar field $a(t)$, depending on time so that

$$
\Delta \mathcal{L}_{a}=\frac{N_{f}}{2 \pi^{2}} K_{\mu} \partial^{\mu} a(x)=\frac{1}{4 \pi^{2}} \mu_{\theta} K_{0} \Longleftrightarrow \mu_{5} \bar{q} \gamma_{0} \gamma_{5} q, \quad \mu_{5} \simeq \dot{a}(t) \simeq \text { const. }
$$

Thus in a quasi-equilibrium situation the appearance of a nearly conserved chiral charge can be incorporated with the help of a axial or a chiral(topological)chemical potential $\mu_{5}$.

\section{Sigma meson model and Wess-Zumino-Witten action in chiral medium}

We will deal only with light scalars mesons. For the description of different properties of low-energy mass spectra of light mesons let us consider a generalized QCD-inspired sigma model with axial chemical potential [10], symmetry under $S U_{L}\left(N_{f}\right) \times S U_{R}\left(N_{f}\right)$ transformation. Further, we will be take into account $u, d$-quarks only,

$$
\begin{aligned}
L= & \frac{1}{4} \operatorname{Tr}\left(D_{\mu} H\left(D^{\mu} H\right)^{\dagger}\right)+\frac{B}{2} \operatorname{Tr}\left[m\left(H+H^{\dagger}\right)\right]+\frac{M^{2}}{2} \operatorname{Tr}\left(H H^{\dagger}\right) \\
- & \frac{\lambda_{1}}{2} \operatorname{Tr}\left[\left(H H^{\dagger}\right)^{2}\right]-\frac{\lambda_{2}}{4}\left[\operatorname{Tr}\left(H H^{\dagger}\right)\right]^{2}+\frac{c}{2}\left(\operatorname{det} H+\operatorname{det} H^{\dagger}\right),
\end{aligned}
$$

where $H=\xi \Sigma \xi$ is an operator for meson fields, $m$ is an average mass of current quarks, $M$ is a "tachyonic" mass generating the spontaneous breaking of chiral symmetry, $B, c, \lambda_{1}, \lambda_{2}$ are real constants (model details see in [10]).

The matrix $\Sigma$ includes the singlet scalar meson $\sigma$, its vacuum average $v$ and the isotriplet of scalar mesons $a_{0}^{0}, a_{0}^{-}, a_{0}^{+}$,

$$
\Sigma=\left[\begin{array}{cc}
v+\sigma+a_{0}^{0} & \sqrt{2} a_{0}^{+} \\
\sqrt{2} a_{0}^{-} & v+\sigma-a_{0}^{0}
\end{array}\right] .
$$

The operator $\xi$ realizes a nonlinear representation of the chiral group and is determined by the isotriplet $\pi^{0}, \pi^{-}, \pi^{+}$of pseudoscalar mesons,

$$
\begin{gathered}
\xi=\exp \left(\frac{i \vec{\pi} \vec{\tau}}{2 f_{\pi}}\right) \approx 1+\frac{i \vec{\pi} \vec{\tau}}{2 f_{\pi}}-\frac{(\vec{\pi} \vec{\tau})^{2}}{8 f_{\pi}^{2}}, \\
\vec{\pi} \vec{\tau}=\left[\begin{array}{cc}
\pi^{0} & \sqrt{2} \pi^{+} \\
\sqrt{2} \pi^{-} & -\pi^{0}
\end{array}\right],
\end{gathered}
$$


where $\vec{\tau}$ are Pauli matrix, $f_{\pi}$ is a decay constant of $\pi$ mesons.

The covariant derivative of $H$ contains external gauge fields $R_{\mu}$ and $L_{\mu}$,

$$
D_{\mu} H=\partial_{\mu} H-i L_{\mu} H+i H R_{\mu}
$$

These fields include the photon field $A_{\mu}$ and are supplemented also a background 4-vector of axial chemical potential $\mu_{5}$,

$$
\begin{aligned}
& R_{\mu}=e Q_{e m} A_{\mu}-\mu_{5} \delta_{\mu, 0} \cdot 1_{2 \times 2}, \\
& L_{\mu}=e Q_{e m} A_{\mu}+\mu_{5} \delta_{\mu, 0} \cdot 1_{2 \times 2},
\end{aligned}
$$

where $Q_{e m}=\frac{1}{2} \tau_{3}+\frac{1}{6} 1_{2 \times 2}$ is a matrix of electromagnetic charge.

Wess-Zumino-Witten action is an important part of effective meson model. It allows us to describe the anomalous decay of strong interaction: $\pi_{0} \rightarrow \gamma \gamma$. Wess-Zumino-Witten action also predicts other meson-photon interactions: $\gamma \pi_{-} \rightarrow \pi_{0} \pi_{-}$and $\gamma \rightarrow \pi \pi \pi$.

We will consider two terms of WZW action: the anomalous pion decay and the vertex of electromagnetic pion form factor. Formula (13) show the view of these terms in non-modified form [11, 12]. After transition to the Sigma-model Lagrangian eigenstates, we obtained innovated vertices, included $a_{0}$ meson decays.

$$
-\frac{e^{2} N_{c}}{24 \pi^{2} f_{\pi}} \epsilon^{\nu \sigma \lambda \rho} \partial_{\sigma} A_{\lambda} \partial_{v} A_{\rho} \pi^{0}-\frac{i e \mu_{5} N_{c}}{6 \pi^{2} f_{\pi}^{2}} \epsilon_{0}^{\sigma \lambda \rho} A_{\rho} \partial_{\sigma} \pi^{+} \partial_{\lambda} \pi^{-}
$$

Here Greek symbols run over one to five. Using this action we can calculate a decay pion or $a$ -meson into two photons.

\section{P-odd vertices for light mesons}

\section{$4.1 \pi^{0} \rightarrow \gamma \gamma$ decay in chiral medium}

It is invited to consider the decay of the neutral pion into two photons. The expression for the differential decay width is given by relation (14). Here $k$ is a momentum of photon and $q$ is a momentum of pion; $\theta$ is angel between $k$ and $q ; f_{-}$is a normalization of wave packets (cf. relation (16)); $C_{+}$is a diagonalization coefficient (cf. [7, 8] ). The connection between $k$ and $q$ is given by expression (15).

$$
\begin{gathered}
\frac{d \Gamma}{d \Omega}=\left(\frac{e^{2} N_{c}}{24 \pi^{2} f_{\pi}}\right)^{2} \cdot \frac{C_{+}^{2}\left(q_{0}\right)|\mathbf{k}|^{3} \sqrt{|\mathbf{k}|^{2}+|\mathbf{q}|^{2}-2|\mathbf{k}||\mathbf{q}| \cos \theta}}{\pi^{2} f_{-}\left(q_{0}\right) \mid 1+\frac{|\mathbf{k}|-|\mathbf{q}| \cos \theta}{\sqrt{|\mathbf{k}|^{2}+|\mathbf{q}|^{2}-2|\mathbf{k}| \mathbf{q} \mid \cos \theta}}} \cdot\left(1+\frac{|\mathbf{k}|-|\mathbf{q}| \cos \theta}{\sqrt{|\mathbf{k}|^{2}+|\mathbf{q}|^{2}-2|\mathbf{k}||\mathbf{q}| \cos \theta}}\right)^{2} \\
|\mathbf{k}|=\frac{m_{e f f+}^{2}\left(\sqrt{|\mathbf{q}|^{2}+m_{\mathrm{eff}+}^{2}}+|\mathbf{q}| \cos \theta\right)}{2\left(m_{\mathrm{eff}+}^{2}+|\mathbf{q}|^{2} \sin ^{2} \theta\right)} \\
f_{-}\left(q_{0}\right)=2 q_{0}+\frac{32 q_{0} \mu_{5}^{2}}{\sqrt{\left(m_{a}^{2}-m_{\pi}^{2}\right)^{2}+\left(8 q_{0} \mu_{5}\right)^{2}}}
\end{gathered}
$$

The $m_{\mathrm{eff}+}$ is effective mass of $a$ meson defined bellow (cf. relation (18)). We briefly discuss the nature of an effective mass. Introduce the definitions for meson state masses in the chiral imbalance 
environment. The mass matrix for scalar and pseudoscalar mesons on the diagonal takes the following values,

$$
\begin{aligned}
& m_{a}^{2}=-2\left(M^{2}-2\left(3 \lambda_{1}+\lambda_{2}\right) v^{2}-c+2 b^{2}\right), \\
& m_{\sigma}^{2}=-2\left(M^{2}-6\left(\lambda_{1}+\lambda_{2}\right) v^{2}+c+2 b^{2}\right), \\
& m_{\pi}^{2}=\frac{2 B m}{v} .
\end{aligned}
$$

After diagonalization we define distorted masses as $m_{e f f+}$ for the field $\tilde{a}$ and $m_{e f f-}$ for the field $\tilde{\pi}$

$$
m_{e f f \pm}^{2}=\frac{1}{2}\left(m_{a}^{2}+m_{\pi}^{2} \pm \sqrt{\left(m_{a}^{2}-m_{\pi}^{2}\right)^{2}+\left(8 b^{\mu} k_{\mu}\right)^{2}}\right) .
$$

The module of photon space momentum is obtained from energy conservation law. The width depends on three variables: space momentum module of photon (or energy of photon on mass-shell), chiral chemical potential and the angle between directions of pion and photon momenta. The width can be integrated over the angle with some boundary values. At zero chiral chemical potential the width value as it known equals to $7.73 \mathrm{eV}$. Our formula gives $7.8 \mathrm{eV}$ at $\mu_{5}$ equal to zero and the angle equal to pi on two ? $\pi / 2$.

The figure 1 (a) shows the set of graphs corresponding to different values of the angle. The chiral chemical potential is equal to $100 \mathrm{MeV}$. As we can see, the width has a maximum that unevenly decreases from 1 to $0,3 \mathrm{eV}$ with the angle increasing. The figure 1 (b) shows the set of graphs for various chemical potential. When it grows by every $100 \mathrm{MeV}$, the width maximum decreases by the order.

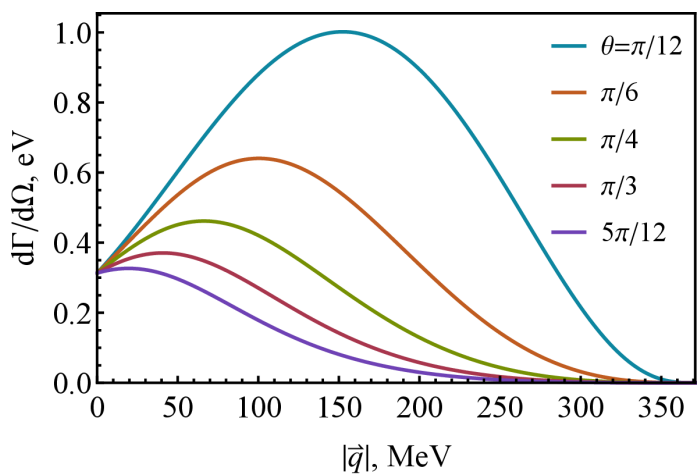

(a)

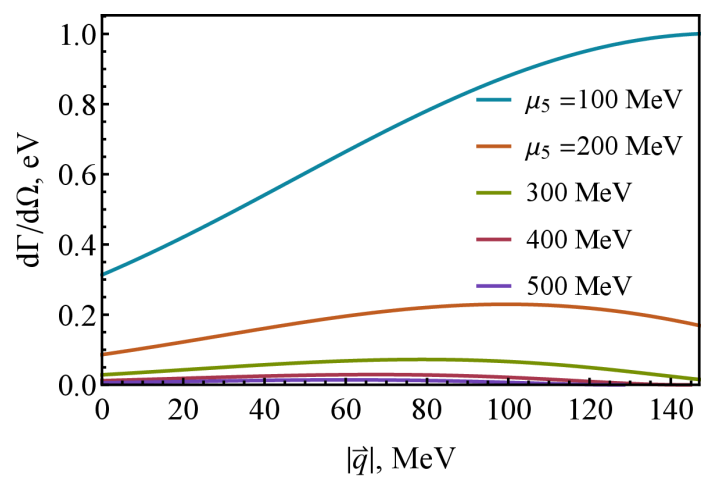

(b)

Figure 1. Differential decay width at $\mu_{5}=100 \mathrm{MeV}$ (a), and $\theta=\pi / 12$ (b)

\section{$4.2 a_{0}^{0} \rightarrow \gamma \gamma$ decay in chiral medium}

Let us consider the decay of neutral $a_{0}$ meson into two photons (cf. figure (2)). This process also arises after mixing of scalar and pseudoscalar mesons from anomalous pion decay. The expression of the differential width has similar view, but its behavior has significant differences. The width monotonically increases with the space momentum module and a chiral chemical potential increasing. The value of this decay width is comparable with the similar decay width of a pion. 


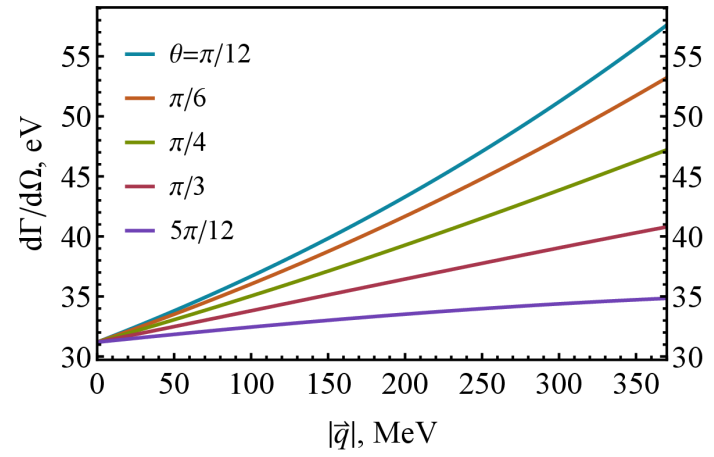

(a)

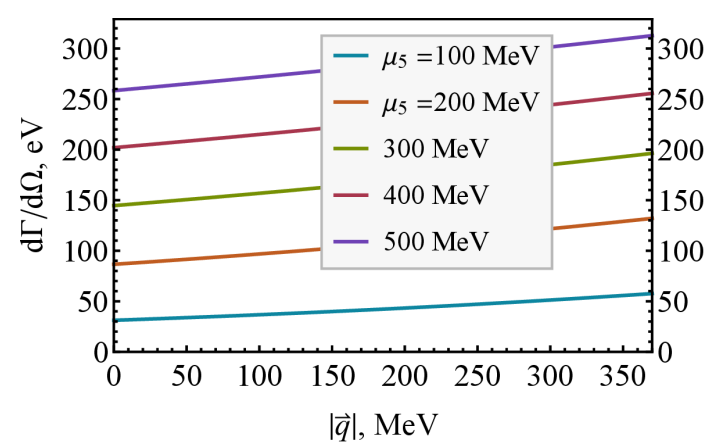

(b)

Figure 2. Differential decay width at $\mu_{5}=100 \mathrm{MeV}$ (a), and $\theta=\pi / 12$ (b)

\section{Pion-photon scattering and asymmetry in photon polarizations}

Let us consider the pion-photon scattering, where the virtual particle can be both a pion and an $a_{0^{-}}$ meson. Two parity-conjugated processes in (19) differ in the polarization of the emitted photons. Due to chiral symmetry violation, an asymmetry of the number of these processes can be observed. The order of asymmetry is such that the effect should be visible on modern accelerators of elementary particles. Therefore, this process could be one of the keys to finding strong CP-violation.

$$
\begin{gathered}
\pi^{ \pm}\left(\vec{p}_{1}\right)+\gamma\left(\vec{k}_{1}\right) \rightarrow \pi^{ \pm}\left(\vec{p}_{2}\right)+\gamma_{+}\left(\vec{k}_{2}\right) \\
\pi^{ \pm}\left(-\vec{p}_{1}\right)+\gamma\left(-\vec{k}_{1}\right) \rightarrow \pi^{ \pm}\left(-\vec{p}_{2}\right)+\gamma_{-}\left(-\vec{k}_{2}\right)
\end{gathered}
$$

Relation (20) is the basic formula of asymmetry in two parity-conjugated processes. The research team of Harada and his colleagues [18] considered the pion-photon scattering with pion as a virtual particle in s-channel (cf. (21)). They obtain interesting expression of asymmetry. There theta is the angle between pion and photon in the final state. In approximation of large energy, zero limit of the angle, value of energy about $1 \mathrm{GeV}$ and chemical potential about $200 \mathrm{MeV}$, the maximum value of the asymmetry is equal to zero point two.

$$
\begin{gathered}
\mathcal{A}=\left|\frac{M_{+}^{2}-\mathcal{P}\left[M_{+}\right]^{2}}{\sum_{m= \pm}\left(M_{m}^{2}+\mathcal{P}\left[M_{m}\right]^{2}\right)}\right| \\
\mathcal{A}^{\text {s-channel }}=\frac{\mu_{5} N_{c}}{12 \pi^{2} f_{\pi}^{2}} \frac{E_{\gamma}\left(E_{\pi}^{2}-m_{\pi}^{2}\right) \sin ^{2} \theta}{m_{\pi}^{2}+E_{\gamma} E_{\pi}\left(1-\sqrt{1-\frac{m_{\pi}^{2}}{E_{\pi}^{2}}} \cos \theta\right)}
\end{gathered}
$$

We assume that at energies of the order of $1 \mathrm{GeV}$, it is necessary to include $a_{0}$ meson as an intermediate particle. For verification of the Ward identity, one needs to consider three diagrams of pion-photon scattering: s-channel, t-channel and four-point vertex (22). For the case of the pion propagator, this identity holds. By transition to modified mesons and adding of resonant $a_{0}$ meson, the identity ceases to be satisfied. Hense we must take into account the contribution from longitudinal polarizations in summation formula. The choice of the chiral chemical potential as a zero component 
of a vector field and the temporal gauge of the electromagnetic field introduce some changes into this relation.

$$
\begin{aligned}
M= & M_{\text {even-even }}+M_{\text {even-odd }} \\
& k_{\mu} M_{\text {even-even }}^{\mu} \neq 0
\end{aligned}
$$

It is invited to consider the general relation (23) for the polarization asymmetry for scattering in s-channel. In the numerator, there are two terms proportional to the first and third order on the chiral chemical potential. The denominator contains four terms of zero, second and fourth order. In the approximation by the first order by $\mu_{5}$ we have the formula similar to the formula, obtained by Harada and his colleagues.

$$
\begin{gathered}
\mathcal{A}^{\mathrm{s} \text {-channel }}=\frac{1}{2} E_{\gamma 2}\left(E_{\pi 2}^{2}-m_{\text {eff- }}^{2}\right) \sin ^{2} \theta_{2} \cdot \\
\cdot\left[C_{n 1} \frac{\mu_{5} N_{c}}{6 \pi^{2} f_{\pi}^{2}} 4\left(E_{\pi 1}^{2}-m_{\text {eff- }}^{2}\right) \sin ^{2} \theta_{1}+C_{n 2}\left(\frac{\mu_{5} N_{c}}{6 \pi^{2} f_{\pi}^{2}}\right)^{3}\left(E_{\pi 1}^{2}-m_{\text {eff- }}^{2}\right) E_{\gamma 1}^{2} \sin ^{2} \theta_{1}\right] \\
\cdot\left[C_{d 1} 16\left(E_{\pi 1}^{2}-m_{\text {eff- }}^{2}\right) \sin ^{2} \theta_{1}\left(E_{\pi 2}^{2}-m_{\text {eff- }}^{2}\right) \sin ^{2} \theta_{2}+\right. \\
+C_{d 2}\left(\frac{\mu_{5} N_{c}}{6 \pi^{2} f_{\pi}^{2}}\right)^{2} 4\left(E_{\pi 1}^{2}-m_{\text {eff- }}^{2}\right) E_{\gamma 1}^{2} \sin ^{2} \theta_{1}\left(E_{\pi 2}^{2}-m_{\text {eff- }}^{2}\right) \sin ^{2} \theta_{2}+ \\
+C_{d 3}\left(\frac{\mu_{5} N_{c}}{6 \pi^{2} f_{\pi}^{2}}\right)^{2} 4\left(E_{\pi 1}^{2}-m_{\text {eff- }}^{2}\right) \sin ^{2} \theta_{1}\left(E_{\pi 2}^{2}-m_{\text {eff- }}^{2}\right) E_{\gamma 2}^{2} \sin ^{2} \theta_{2}+ \\
\left.+C_{d 4}\left(\frac{\mu_{5} N_{c}}{6 \pi^{2} f_{\pi}^{2}}\right)^{4}\left(E_{\pi 1}^{2}-m_{\text {eff- }}^{2}\right) E_{\gamma 1}^{2} \sin ^{2} \theta_{1}\left(E_{\pi 2}^{2}-m_{\text {eff- }}^{2}\right) E_{\gamma 2}^{2} \sin ^{2} \theta_{2}\right]^{-1}
\end{gathered}
$$

We can consider one of the coefficients of the numerator. The expression is sown by the formula (24). It consist of three terms corresponding to different combinations of propagators and different types of amplitudes. The first describes combination of two amplitudes with pion propagators. The second describes term with two resonant $a_{0}$ meson propagator. And the last two terms has common factor, combining both a pion and an $a_{0}$ meson propagators. In every term, one of the amplitudes is even and another is odd. 


$$
\begin{gathered}
C_{n 1}=\frac{4 p_{0}^{\prime 2} / f_{-}^{\prime 2}}{\left(p^{\prime 2}-m_{\mathrm{eff}}^{2}\left(p_{0}^{\prime}\right)\right)^{2}} \cdot C_{+}\left(p_{0}^{\prime}\right) C_{+}\left(E_{\pi 2}\right) \cdot \\
\cdot\left(C_{+}\left(E_{\pi 1}\right) C_{+}\left(p_{0}^{\prime}\right)+C_{-}\left(E_{\pi 1}\right) C_{-}\left(p_{0}^{\prime}\right)\right)^{2}\left(C_{+}\left(E_{\pi 2}\right) C_{+}\left(p_{0}^{\prime}\right)+C_{-}\left(E_{\pi 2}\right) C_{-}\left(p_{0}^{\prime}\right)\right)+ \\
+\frac{4 p_{0}^{\prime 2} / f_{+}^{\prime 2}}{\left(p^{\prime 2}-m_{\mathrm{eff}+}^{2}\left(p_{0}^{\prime}\right)\right)^{2}+m_{\mathrm{eff}+}^{2}\left(p_{0}^{\prime}\right) \Gamma_{a}^{2}} \cdot C_{-}\left(p_{0}^{\prime}\right) C_{+}\left(E_{\pi 2}\right) \cdot \\
\cdot\left(C_{+}\left(E_{\pi 1}\right) C_{-}\left(p_{0}^{\prime}\right)-C_{-}\left(E_{\pi 1}\right) C_{+}\left(p_{0}^{\prime}\right)\right)^{2}\left(C_{+}\left(E_{\pi 2}\right) C_{-}\left(p_{0}^{\prime}\right)-C_{-}\left(E_{\pi 2}\right) C_{+}\left(p_{0}^{\prime}\right)\right)+ \\
+\frac{\left(p^{\prime 2}-m_{\mathrm{eff}+}^{2}\left(p_{0}^{\prime}\right)\right) 4 p_{0}^{\prime 2} / f_{-}^{\prime} f_{+}^{\prime}}{\left(p^{\prime 2}-m_{\mathrm{eff}-}^{2}\left(p_{0}^{\prime}\right)\right)\left(\left(p^{\prime 2}-m_{\mathrm{eff}+}^{2}\left(p_{0}^{\prime}\right)\right)^{2}+m_{\mathrm{eff}+}^{2}\left(p_{0}^{\prime}\right) \Gamma_{a}^{2}\right)} \cdot \\
\cdot\left(\left(C_{+}\left(E_{\pi 1}\right) C_{+}\left(p_{0}^{\prime}\right)+C_{-}\left(E_{\pi 1}\right) C_{-}\left(p_{0}^{\prime}\right)\right)\left(C_{+}\left(E_{\pi 2}\right) C_{+}\left(p_{0}^{\prime}\right)+C_{-}\left(E_{\pi 2}\right) C_{-}\left(p_{0}^{\prime}\right)\right) .\right. \\
\cdot\left(C_{+}\left(E_{\pi 1}\right) C_{-}\left(p_{0}^{\prime}\right)-C_{-}\left(E_{\pi 1}\right) C_{+}\left(p_{0}^{\prime}\right)\right) C_{-}\left(p_{0}^{\prime}\right) C_{+}\left(E_{\pi 2}\right)+ \\
+\left(C_{+}\left(E_{\pi 1}\right) C_{-}\left(p_{0}^{\prime}\right)-C_{-}\left(E_{\pi 1}\right) C_{+}\left(p_{0}^{\prime}\right)\right)\left(C_{+}\left(E_{\pi 2}\right) C_{-}\left(p_{0}^{\prime}\right)-C_{-}\left(E_{\pi 2}\right) C_{+}\left(p_{0}^{\prime}\right)\right) . \\
\left.\left.\cdot\left(C_{+}\left(E_{\pi 1}\right) C_{+}\left(p_{0}^{\prime}\right)+C_{-}\left(E_{\pi 1}\right) C_{-}\left(p_{0}^{\prime}\right)\right) C_{+}\left(p_{0}^{\prime}\right)\right) C_{+}\left(p_{0}^{\prime}\right) C_{+}\left(E_{\pi 2}\right)\right)
\end{gathered}
$$

Figure 3 shows a view of the polarization asymmetry with energy equal to $1 \mathrm{GeV}$ and chemical potential equal $200 \mathrm{MeV}$. The maximum value of the asymmetry is about zero point two.

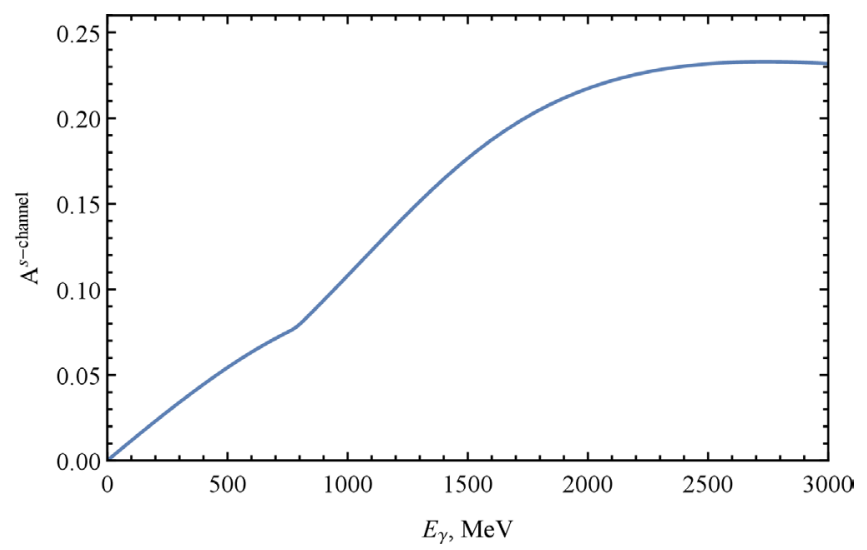

Figure 3. Asymmetry, $\mu_{5}=200 \mathrm{MeV}, E_{\pi 2}=1 \mathrm{GeV}$

\section{Conclusions and outlook}

In this work we described a possibility of Local Parity Breaking (LPB) emerging in a dense hot baryon matter (hadron fireball) in heavy-ion collisions (HIC) at high energies. To describe such a quark-hadron matter in fireball chiral chemical potential can be introduced to quantify the presence of Chirality Imbalance i.e. the difference between the average numbers of right and left- handed quarks in the fireball after HIC. More precisely the phenomenology of LPB in the fireball induces the introduction of a topological charge and, accordingly, a topological (chiral) chemical potential. Topological charge fluctuations transmit their influence to hadronic physics via an axial chemical potential. Based on 
the effective meson Lagrangian motivated by QCD in the chiral medium the properties of light scalar and pseudoscalar mesons are analyzed. It is establish that exotic decays of scalar mesons arise as a result of mixing of $\pi$ and $a_{0}$ vacuum states in the presence of chiral imbalance. The pion electromagnetic formfactor obtains a parity-odd supplement which generates a photon polarization asymmetry in pion polarizability. We consider that the above-mentioned properties of LPB can be revealed in experiments on LHC, RHIC, CBM FAIR and NICA accelerators

In addition, we already suggested the vector meson dominance model [1], with chiral imbalance: the spectrum of massive vector mesons splits into three components with different polarizations and with different effective masses that can be used to detect local parity breaking. The proposed schemes for revealing local parity breaking helps to (partially) explain qualitatively and quantitatively the anomalous yield of dilepton pairs in the CERES, PHENIX, STAR, NA60, and ALICE experiments. Accordingly, the identification of its physical origin might serve as a base for a deeper understanding of QCD properties in a medium under extreme conditions.

\section{Acknowledgements}

It is a pleasure to thank the organizers of the XXth International Seminar "Quarks-2018" for a fruitful meeting and an excellent atmosphere. This work has been supported through grants FPA2013-46570, 2014-SGR-104 and Consolider CPAN. Funding was also partially provided by the Spanish MINECO under project MDM-2014-0369 of ICCUB (Unidad de Excelencia 'Maria de Maeztu'). A.A. and V.A. were supported by Grant RFBR projects 16-02-00348 and also got a financial support of SPbSU, in particular, by projects Grant id=27800514; 27803510; 27944831.

\section{References}

[1] A.A. Andrianov, V.A. Andrianov, D. Espriu, X. Planells, Phys. Lett. B 710, 230 (2012); Phys. Rev. D 90, 034024 (2014).

[2] I. Tserruya, Landolt-Börnstein 23, 176 (2010).

[3] A.A. Andrianov, V.A. Andrianov, D. Espriu, X. Planells, Theor. Math. Phys. 170, 17 (2012); A.A. Andrianov, V.A. Andrianov, Theor. Math. Phys. 185, 1370 (2015).

[4] A. B. Migdal, Zh. Eksp. Teor. Fiz., vol. 61,2210 (1971).

[5] D. Kharzeev, R. D. Pisarski, and M. H. G. Tytgat, Phys. Rev. Lett. 81, 512 (1998).

[6] K. Buckley, T. Fugleberg and A. Zhitnitsky, Phys. Rev. Lett. 84, 4814 (2000).

[7] A. A. Andrianov, V. A. Andrianov, D. Espriu, A. E. Putilova, A. V. Iakubovich, in Acta Physica Polonica B, Proceedings Supplement, vol. 10, pp. 977-982 (2017).

[8] A. A. Andrianov, V. A. Andrianov, D. Espriu, A. V. Iakubovich, A. E. Putilova, EPJ Web Conf. vol. 158, 10, (2017).

[9] A. Yamamoto, Phys. Rev. D 84, 114504 (2011); A. Yamamoto, Phys. Rev. D 107, 031601 (2011).

[10] A. A. Andrianov, D. Espriu and X. Planells, Eur. Phys. J. C 73:2294 (2013).

[11] J. Wess and B. Zumino, Phys. Lett. 37B, 95 (1971); E. Witten, Nucl. Phys. B 223, 422 (1983)

[12] A. A. Andrianov, V. A. Andrianov, V. Yu. Novozhilov, and Yu. V. Novozhilov, Theor. Math. Phys., 70, 43 (1987).

[13] A. B. Migdal, Zh. Eksp. Teor. Fiz., vol. 61 pg. 2210 (1971).

[14] T. D. Lee and G. C. Wick. Phys. Rev. D 9, 2291 (1974)

[15] A.A. Andrianov, D. Espriu, V.A. Andrianov, Phys. Lett. B, vol. 678, Issue 4, 416-421 (2009). 
[16] D. Kharzeev, R. D. Pisarski, and M. H. G. Tytgat, Phys. Rev. Lett. 81, 512 (1998).

[17] K. Buckley, T. Fugleberg and A. Zhitnitsky, Phys. Rev. Lett. 84, 4814 (2000).

[18] Kawaguchi M., Harada M., Matsuzaki S., Ouyang R.M., Phys. Rev. C 95, 065204 (2017) .

[19] N. Ajitanand, S. Esumi, R. Lacey, P. Collaboration, et al., P-and CP-odd effects in hot and dense matter, in: Proc. of the RBRC Workshops, Vol. 96, 2010.

[20] P. Wurn et al. (CERES Collab.), Nucl. Phys. A, 5901-2, 103-116 (1995); CERES Collaboration (Agakichiev, G. et al.) Phys. Rev. Lett. 75 , 1272 (1995); Phys. Lett. B 422, 405(1998); Eur. Phys. J. C 41, 475(2005).

[21] A.A. Andrianov, V.A. Andrianov, D Espriu, A.E. Putilova, A.V. Iakubovich, Phys. Part. Nuclei Lett. 15: 357, (2018).

[22] A.A. Andrianov, D. Espriu, V.A. Andrianov, Phys. Lett. B, vol. 678, Issue 4, 416-421 (2009). 